\title{
SUBJETIVIDADE MASCULINA E O CUIDADO EM SAÚDE NA ATENÇÃO PRIMÁRIA
}

Tatieli Dagostim PAIM

Thiago REGINALDO ${ }^{2}$

Silvio Serafim da Luz FILHO ${ }^{3}$

${ }^{1}$ Mestranda em Engenharia e Gestão do Conhecimento (UFSC - PPGEGC); Especialista em Terapia Intensiva (INSPIRAR) e em Ciências dos Saberes da Educação (UNIBAVE); Graduada em Fisioterapia (UNESC). tatielidpaim@gmail.com

${ }^{2}$ Mestrando em Engenharia e Gestão do Conhecimento (UFSC - PPGEGC); Especialista em Comunicação e Marketing em Mídias Digitais (Estácio de Sá); Graduado em Design (UFSC), Gestão da Tecnologia da Informação (IFSC) e Educação Física (UDESC); graduando em Pedagogia (UNISUL). thiagoreginaldo@ yahoo.com.br

${ }^{3}$ Docente da Universidade Federal de Santa Catarina (UFSC) do Programa de Pós-Graduação em Engenharia e Gestão do Conhecimento (PPGEGC). Doutor em Educação (USP); Mestre em Administração (UFRGS); Especialista em Psicologia Escolar (PUCRS) e em Orientação Educacional (UDESC); Graduado em Psicologia (PUC/PR). silvioserafim@bol.com.br

\section{Recebido em: 19/05/2014 - Aprovado em: 30/06/2014 - Disponibilizado em: 30/07/2014}

RESUMO: As mudanças histórico-sociais do comportamento humano são responsáveis pelo surgimento de definições de masculinidade em aspectos de sentido e significado. Os diversos meios de comunicação atuais tratam desse assunto no intuito de delinear o contorno daquele que no fundo é um desconhecido - o homem. No sentido de fomentar essa discussão, e compreender esse homem subjetivo e sua saúde, analisaram-se as informações recorrentes em periódicos científicos sobre a subjetividade masculina com o cuidado em saúde na atenção primária. Para tanto utilizou nos seus procedimentos metodológicos o processo de busca sistemática para poder refletir sobre a perspectiva relacional de gênero e sexualidade, hegemonia masculina, presença ou não de barreiras institucionais de serviços e da população masculina nos serviços de saúde. Verificou-se a necessidade de mudanças na forma de pensar tanto nos profissionais de saúde como na população masculina para que os cuidados à saúde sejam vistos como necessidades básicas a esta população.

Palavras chave: Saúde Masculina. Subjetividade Masculina. Atenção primária. Gênero. Sexualidade.

\section{MASCULINE SUBJECTIVITY AND THE HEALTH CARE IN PRIMARY CARE}

\begin{abstract}
The social and historical changes in human behavior are responsible for the emergence of definitions of masculinity in aspects of meaning and significance. The various current communication medium this matter in order to delineate the outline of that which is in fact a stranger - the man. In order to foster this discussion, and understand this subjective man and his health, was analyzed the information in scientific journals on the masculine subjectivity with health care in primary care. For both methodological procedures used in the systematic search process to be able to reflect on the relational perspective of gender and sexuality, male hegemony, presence or absence of institutional barriers to services and of the male population in health services. There is a need to change the way of thinking in both health professionals as the male population so that health care are seen as basic needs of this population.
\end{abstract}

Keywords: Men's Health. Masculine Subjectivity. Primary care. Genre. Sexuality.

\section{INTRODUÇÃO}

Ao se pensar no ser humano em uma abordagem clínica Focault (1998) afirma que o corpo apresenta por direito de natureza o espaço de origem e a repartição da doença.
No entanto este espaço de corpo é somente uma das maneiras da saúde abordar o ser humano. A subjetividade está presente nas esferas de geração de sentido e significado deste ser e sua compreensão é uma aliada na 
representação da saúde. Estreitando-se ainda mais o foco da saúde no homem, ser masculino, outros pontos revelam novos desafios para este século no que tange ao seu corpo e subjetividade ligados a saúde.

As mudanças de comportamento e a crise na identidade masculina estão favorecendo o surgimento de um "novo homem", a mídia, e principalmente os cadernos de comportamentos de grandes jornais têm tratado com insistência esses assuntos na busca em delinear os contornos daquele que no fundo ainda é um desconhecido (CALDAS, 1997). Como exemplos atuais dessas mídias existem as revistas especializadas em saúde masculina, páginas em plataformas digitais, campanhas e políticas no portal de saúde do governo brasileiro, entre outras. No caso dos portais e campanhas públicas de saúde torna-se necessário compreender como a saúde masculina é encarada pelos serviços de saúde, principalmente os de atenção primária, em relação a sua subjetividade. Este artigo tem este objetivo ao analisar a relação da subjetividade masculina com o cuidado em saúde na atenção primária por meio de uma busca sistemática qualitativa. Os cuidados de saúde em atenção primária são os responsáveis pela prevenção de doenças e promoção da saúde.

\section{JORNADA METODOLÓGICA}

$\mathrm{O}$ tipo de pesquisa que se realizou neste artigo é caracterizado como uma busca sistemática que é uma das etapas de uma revisão sistemática. Isso foi feito para eleger os artigos a serem discutidos a partir de uma abordagem qualitativa. Usou de métodos explícitos e reprodutíveis a partir de uma visão geral de estudos primários. Os métodos explícitos ajudam a limitar o viés e rejeitar estudos, as conclusões são mais confiáveis e precisas, grandes quantidades de informações podem ser assimiladas pelos profissionais da saúde e demais pesquisadores, os resultados da pesquisa podem estabelecer uma generalização e consistência de informações e identificar inconsistências (GREENHALGH, 1997).

Foi conduzida uma pesquisa da literatura publicada de 1960 até 15 de setembro de 2013, aonde foram usadas as seguintes bases de dados: Scopus e Scielo. A pesquisa foi limitada a artigos ou revisões com as seguintes palavras chave nos títulos: saúde masculina, subjetividade masculina e atenção primária. Foram encontrados artigos em português e inglês. Esses termos foram posteriormente reduzidos e se excluíram os que discutiam sobre mulheres, comunidade GLBTTTI (Gays, Lésbicas, Bissexuais, Travestis, Transexuais, Transgêneros e Intersexuais), estudos animais e tópicos muito específicos de determinada região geográfica. Isso resultou num total de 17 artigos dos 312 da Scopus e 46 da Scielo. A partir dos 17 artigos foi feita uma abordagem qualitativa de 
discussão da temática de saúde masculina sobre os seus aspectos de subjetividade, gênero e sexualidade, e os programas de prevenção e promoção de saúde na atenção básica.

\section{DISCUSSÃO DOS ARTIGOS}

Percebe-se que uma hegemonia foi criada ao longo do tempo em torno da masculinidade e que isso tem influências na saúde do homem. Portanto, cabe nessa discussão compreender o que está sendo estudado nessa área e quais são os principais resultados. Isso aconteceu por meio de uma revisão de periódicos científicos que tratam da subjetividade masculina com o cuidado em saúde na atenção primária.

Um dos primeiros motivos para se pesquisar sobre saúde masculina é o fato dos homens morrerem mais que as mulheres em todas as faixas etárias e mais cedo (BRAZ, 2005; FIGUEIREDO, 2005; CARRARA et al., 2009; SEPARAVICH \& CANESQUI; GOUGH, 2013; EVANS, 2011). A sobrevida da mulher sempre foi maior com uma média de 7,8 anos a mais, além disso, a mortalidade masculina nos jovens e adultos jovens é justificada pelas suas identidades comportamentais violentas com um estilo de vida de alto risco (álcool, cigarro, estresse, trabalho) e pela sua concepção de invulnerabilidade as doenças (BRAZ, 2005; FIGUEIREDO, 2005; CARRARA et al., 2009; GOUGH, 2013; EVANS, 2011).
Alguns estudiosos demonstram que ser do sexo masculino é fator determinante de morte prematura, a faixa etária de sobre mortalidade neste gênero é durante os 20 anos e após os 60 anos (BRAZ, 2005; EVANS, 2011). A mortalidade masculina é 50\% maior do que a feminina (SEPARAVICH \& CANESQUI, 2013). Os fatores externos são os preponderantes para a mortalidade, acidentes de transportes, as lesões auto provocadas, as agressões e homicídios, foram responsáveis por $78 \%$ de mortes em 2005 no Brasil (SEPARAVICH \& CANESQUI, 2013; FIGUEIREDO, 2005).

Como justificativa dessa mortalidade maior dos homens em relação às mulheres está o fato delas realizarem mais consultas e abordarem suas doenças com mais facilidade, em contrapartida os homens procuram atendimentos principalmente em emergências e farmácias quando estão com problemas graves e dores insuportáveis que os impedem de trabalhar e realizar suas atividades (BRAZ, 2005; CARRARA et al., 2009) e quando necessitam de internação hospitalar estão em situações graves e permanecem por longos períodos internados (BRAZ, 2005). Quanto à morbidade, as internações mais frequentes são por tumores e causas externas, onde os casos de neoplasias são do aparelho respiratório, digestivo e urinário, sendo que o câncer de próstata sobressai os demais, uma variação de $195,3 \%$ e $502 \%$ de custos ao Sistema Único de Saúde (SUS) (CARRARA et al., 2009). Os 
exames preventivos poderiam descobrir essas enfermidades precocemente e o tratamento poderia ser mais efetivo (Ibid.).

Ao se pensar nessa relação do homem em um contexto histórico de procura aos serviços de saúde prevalece mais uma vez o foco na atenção a saúde da mulher. Em 1930 as medidas especiais à saúde das mulheres já eram nítidas no Brasil, focalizadas na dicotomia materno-infantil; em 1983 houve então a criação do PAISM (Programa de Atenção Integral à Saúde da Mulher) implantado em todos os serviços de saúde do país com objetivo de direcionar as mulheres a compreensão do seu corpo com ênfase na sua singularidade relacionado à sua vida sexual e reprodutiva ((FIGUEIREDO, 2005; SEPARAVICH \& CANESQUI, 2013). Em 2007, 25 anos depois da implantação do programa direcionado a saúde feminina, José Gomes Temporão, o Ministro da Saúde daquele ano, sugeriu a implantação de uma política nacional para atender as necessidades de saúde do homem (FIGUEIREDO, 2005). Em 2008 foi criada, a primeira edição da Política Nacional de Atenção Integral a Saúde do Homem (PNAISH), pelo Departamento de Ações Programáticas Estratégicas da Secretaria de Atenção à Saúde, a Área Técnica de Saúde do Homem, o que tornou o Brasil o segundo país da América a apresentar um setor especifico para o homem, o Canadá foi o pioneiro, deixando para trás os Estados Unidos da América (Ibid.).
A criação desta política teve como finalidade a promoção da assistência ao homem no Sistema Único de Saúde (SUS) e a redução das taxas de morbimortalidade masculina (FIGUEIREDO, 2005; SEPARAVICH \& CANESQUI, 2013). Em 28 de agosto de 2009, no auditório da Organização Pan-Americana de Saúde em Brasília, o Ministério da Saúde decreta oficialmente a Política (PNAISH) para valorização do homem brasileiro (FIGUEIREDO, 2005). Após a implantação desta política pública direcionada a saúde dos homens, além das mídias veiculadas na imprensa, a saúde coletiva foi abordada não apenas para sexualidade masculina, mas direcionada para as contribuições da perspectiva de gênero e subjetividades com o compromisso de desenvolver inovações em serviços e entender as necessidades para os programas e ações de saúde (ROBINSON \& ROBERTSON, 2010).

Mesmo com todos os indícios sobre a taxa de mortalidade e morbidade elevados e o fator de risco "homem" ser preponderante a sociedade demorou quase 25 anos para implantar um programa em prol da saúde direcionada a esta população. Assim, a saúde masculina deve ser entendida em seus comportamentos de "gênero" e em seus fatores biológicos de "sexualidade".

O gênero é um elemento característico das relações sociais, demonstra as diferenças sócio-comportamentais desenvolvidas entre 
homens e mulheres, e os significados de poder entre eles. É considerado um fator da morbimortalidade pela sua identidade característica masculina, isto é, alta exposição a riscos e a falta de cuidados em saúde (FIGUEIREDO, 2005; SEPARAVICH \& CANESQUI, 2013; NÄSLINDHYLISPANGAR et al., 2008; EVANS, 2011; LOHAN, 2007). A dificuldade em equilibrar a saúde e o bem estar acontece devido a um forte padrão institucionalizado de masculinidade, visto que, muitos homens justificam suas falhas na sua identidade de gênero e esquecem sua identidade pessoal (BRAZ, 2005; GOUGH, 2013). Para melhor compreender a categoria gênero e sexualidade na identidade masculina aprofunda-se a discussão sobre sua subjetividade.

\section{Subjetividade masculina: gênero e sexualidade}

O processo de construção da subjetividade ocorre desde a concepção do individuo até a vida adulta viril, perpassando pelos fatores psicossociais, biofísicos e culturais (BRAZ, 2005; SMITH, 2007). Os aspectos que influenciam na subjetividade são o sexo (fator biológico determinado) e o gênero (fator comportamental variável) (EVANS, 2011). A masculinidade está relacionada com a perspectiva de gênero onde os comportamentos socioculturais oferecem o conceito a sua identidade.

Os homens expressam sua masculinidade no corpo e no comportamento, são criados para serem fortes, protetores e viris; treinados a suportar as dores físicas e emocionais, já que a dor os iguala as mulheres, e direcionam fortes padrões de comportamentos como a prática de esportes violentos com o objetivo de diferenciar-se da mulher (BRAZ, 2005; GOMES, 2003). Isso dificulta o seu processo de auto cuidado, ou seja, de procurar assistência, já que foram orientados e exigidos a cuidar de sua família e não de si próprios (Ibid.).

A identidade masculina foi criada na sociedade por meio da metáfora "ser homem de verdade é..." um indivíduo reservado no âmbito pessoal, solitário, prático, objetivo e caso constitua uma família ser seu guardião protetor. Eles se adéquam aos modelos tradicionais hegemônicos da sociedade como agressivos e expressam medo quando questionados de suas masculinidades ao agirem por estereótipos, e como consequência deixam sua identidade pessoal pela de gênero e justificam a pressão social imposta (GOMES, 2003; SCHRAIBER et al., 2010; CARRARA et al., 2009; SEPARAVICH \& CANESQUI, 2013; GOUGH, 2013; CRAWSHAW, 2007; FILHO et al., 2012; ROBINSON \& ROBERTSON, 2010).

A subjetividade masculina é influenciada pelas identidades hegemônicas e neste ponto são percebidas como práticas de gênero socialmente aceitas num sentido ideológico marxista. Então firma-se uma hegemonia tradicional: ser branco, 
heterossexual, classe média, traços de assertividade, dominação, controle, força psicológica e restrição emocional (EVANS, 2011, LOHAN, 2007). Todavia apesar desses padrões institucionalizados o ser humano se difere em suas masculinidades entre as sociedades e na mesma sociedade em períodos históricos distintos (SEPARAVICH \& CANESQUI, 2013). Numa leitura Junguiana, os homens buscam se autoafirmar e se sobressair sobre as mulheres, vendo como natural serem agressivos, provedores e competitivos. A questão não é apenas da cultura, é como o homem se insere nela, suas questões sociais e históricas, para isso o entendimento de gênero se demonstra efetivo, pois, se deve compreender que cada ser é singular e merece ser tratado individualmente (NÄSLINDH-YLISPANGAR et al., 2008; GRIFFITH, 2012).

A compreensão desta subjetividade masculina para os homens e profissionais da saúde auxilia no entendimento de que existem muitos modelos presentes e estes devem ser vistos social e historicamente durante a realização de promoções e serviços de saúde.

\section{Programas de prevenção e promoção de saúde na atenção básica}

A falta de equidade nos serviços direcionados a saúde é observada pela visão social de que o homem não se cuida. Assim, a mulher é considerada frágil e o homem invulnerável às doenças com a presença de serviços de saúde abundantes para mulheres e crianças, e o fortalecimento de ausências desses serviços para a população masculina (BRAZ, 2005; GOMES, 2003; FIGUEIREDO, 2005).

A mudança da saúde relacionada na perspectiva de gênero ocorrerá quando houver uma transformação na mentalidade da sociedade como um todo, homens, mulheres, mas principalmente dos profissionais e administradores destes serviços de saúde (BRAZ, 2005) para compreenderem os sentidos simbólicos envolvidos (GOMES, 2003).

Os profissionais de saúde consideram os homens como curativos, pois, só procuram os serviços quando apresentam doenças graves, entretanto, esta construção cultural do adoecimento e cuidado é atribuída não só aos homens, mas aos profissionais que operam os mesmos às reduções biomédicas. O problema é que os profissionais não entendem o homem quanto a sua complexidade e sim como patologias anatômicas e fisiológicas (SEPARAVICH \& CANESQUI, 2013). Deste modo, direcionam apenas medicações e esquecem sua subjetividade, tornam-se neutros nos atendimentos e em alguns casos por não visualizarem seus problemas reais deslocam os homens para o lado de fora dos serviços de atenção primária (SCHRAIBER et al., 2010).

A ausência masculina nos serviços de saúde pode ser justificada por dois fatores, as barreiras institucionais, que são as 
dificuldades de acesso aos serviços de saúde, e as barreiras socioculturais, quando o homem considera-se invulnerável as doenças, pois, ficar doente para ele demonstra fragilidade masculina (FIGUEIREDO, 2005; CARRARA et al., 2009). A hegemônica tradicional masculina, apesar de estar se modificando, ainda prevalece no âmbito da saúde na perspectiva relacional do gênero devido à identidade masculina preponderante, como forte e viril (Ibid.).

Os homens procuram os serviços apenas quando estão doentes, com alguma dor, devido as suas ausências no trabalho, medo de perder o emprego, demora no atendimento público, receio que suas informações de saúde sejam passadas para outras pessoas, que não o médico (NÄSLINDH-YLISPANGAR et al., 2008; NOONE \& STEPHENS, 2008; CRAWSHAW, 2007; KRAHN \& OUTRAM, 2012; ROBINSON \& ROBERTSON, 2010).

Os homens não tomam medidas preventivas devido à falta de tempo ou por não apresentarem nenhum sintoma, se sentirem saudáveis e até mesmo preguiça (Ibid.). Eles conseguem expressar seus sintomas e sentimentos, mas não o fazem com medo de serem ridicularizados pela sociedade devido à presença da masculinidade (Ibid.). Não querem ser dependentes de outros homens e mulheres para assisti-los e não procuram serviços de saúde porque não querem igualdade com as mulheres (Ibid.).
A relação entre o profissional de saúde e o paciente homem deve ocorrer de maneira detalhada para entender seus significados diante do processo saúde-doença. Muitos homens apresentam receio em procurar instituições de saúde por medo de encontrarem alguma patologia e pelo posicionamento "neutro" do profissional em atendê-lo. Estes pacientes não têm assiduidade nos serviços, então quando o buscam, apresentam inúmeras dúvidas e necessidades que os afligem, portanto esperam que os profissionais saneiem suas interrogativas dentro de sua identidade masculina. Assim, justifica-se a formação mais social desses profissionais de saúde no gerenciamento de programas de prevenção e promoção de saúde para atender as premissas que foram deixadas para trás por estes indivíduos em toda a sua existência histórica, cultural e social.

\section{CONSIDERAÇÕES FINAIS}

Através da busca sistemática foi possível delinear a discussão sobre a subjetividade masculina relacionada aos serviços de saúde na atenção primária. Com os resultados é importante destacar que a relação de subjetividade masculina na saúde está ligada ao contexto sócio histórico inserido, onde o fator fundamental esta na construção enquanto perspectiva de gênero, ou seja, os fatores de variáveis comportamentais na construção de sentido e 
significado masculino. Nota-se que o padrão hegemônico que foi institucionalizado ao longo dos tempos, como o comportamento do homem se portar diante das coisas, influencia na procura pelos serviços de saúde. O homem se porta como um indivíduo que domina, tem força psicológica, restrição emocional, agressivo, viril, competitivo, entre outras características que contribuem na construção de um estereótipo. Essas características de gênero masculino podem dificultar a compreensão do que realmente é este ser humano por trás dessa moldura social e quais são suas reais necessidades na procura de um serviço de saúde.

Houve um avanço no elo entre homem e serviços de saúde, após a implantação da Política Nacional de Atenção Integral à Saúde do Homem nos sistemas públicos. A Agência Nacional de Saúde, que regulamenta os planos de saúde privados, também seguiu o mesmo caminho e abordou recentemente os programas de prevenção como fatores diferenciais e de pontuações para os planos. Entretanto, mesmo com essas iniciativas públicas e privadas precisa-se voltar à atenção para o conhecimento dos profissionais de saúde na subjetividade masculina, para almejar maior assiduidade do homem nos serviços de saúde e promover sua qualidade de vida e bem estar.

A partir do momento em que as pessoas compreenderem melhor a subjetividade masculina enquanto perspectiva de gênero poderá aparecer muitos benefícios nos serviços e programas de saúde. Dentre eles podem ser oferecidos maior disponibilidade de horários e mudança no ambiente com enfoque direcionado aos homens. Por exemplo, nos locais de atendimento presenciais poderia haver cartazes e folders voltados a este público. Já nas mídias digitais poderiam ser feitas campanhas de saúde masculina com mais frequência e com diferentes enfoques numa atenção integral de gênero ligada a saúde e prevenção. A partir de iniciativas como essas podem começar mudanças nos sujeitos e consequentemente na prestação dos serviços de saúde e atendimento a população masculina. Assim, os homens passam a compreender que eles são tão importantes quanto às mulheres e que precisam de cuidados. Passam também a se observarem melhor dentro de suas masculinidades, realizam exames preventivos sem preconceitos e até mesmo desenvolvem um tratamento com mais eficiência. O "tabu" homem nos serviços de saúde pode ser modificado pela informação e conhecimento de que o padrão hegemônico, estereótipos e outras couraças sociais atrapalham o bem estar e sua subjetividade. 


\section{REFERÊNCIAS}

BRAZ, M. A construção da subjetividade masculina e seu impacto sobre a saúde do homem: reflexão bioética sobre justiça distributiva. Ciência saúde coletiva 10(1): 97-104, 2005, Março.

CALDAS, D. Homens: comportamento, sexualidade, mudança. São Paulo: SENAC, 1997.

CARRARA, S.; RUSSO, J. A.; FARO, L. A política de atenção à saúde do homem no Brasil: Os paradoxos da medicalização do corpo masculino. Physis, 19(3), 659-678, 2009.

CRAWSHAW, P. Governing the healthy male citizen: Men, masculinity and popular health in men's health magazine. Social Science and Medicine, 65(8), 1606-1618, 2007.

EVANS, J.; FRANK, B., OLIFFE, J. L.; GREGORY, D. Health, illness, men and masculinities (HIMM): A theoretical framework for understanding men and their health. Journal of Men's Health, 8(1), 7-15, 2011.

FIGUEIREDO, W. Assistência à saúde dos homens: um desafio para os serviços de atenção primária. Ciência \& Saúde Coletiva, 10(1): 105-109, 2005.

FILHO, E. P. S.; NETO, M. L. R.; TEIXEIRA, S. A. Masculine subjectivity as a challenge for men's health.Health. MED, 6(7), 2435-2437, 2012.

FOCAULT, M. O nascimento da clínica. 5. ed. Rio de Janeiro: Forense Universitária, 1998.

GOMES, R. Sexualidade masculina e saúde do homem: proposta para uma discussão. Ciência saúde coletiva, 8(3): p. 825-829, 2003.

GOUGH, B. The psychology of men's health: Maximizing masculine capital. Health
Psychology, 32(1), p. 1-4, 2013.

GREENHALGH, T. Papers that summarise other papers (systematic review and metaanalyses). BMJ. Sep; 13(315):672-5, 1997.

GRIFFITH, D. M. An intersectional approach to men's health. Journal of Men's Health, 9(2), p. 106-112, 2012.

KRAHN, T. M.; OUTRAM, S. "Go now": A popular film showing the potential for "masculinity" to facilitate health-promoting behaviours. International Journal of Men's Health, 11(2), p. 107-126, 2012.

LOHAN, M. How might we understand men's health better? integrating explanations from critical studies on men and inequalities in health. Social Science and Medicine, 65(3), p. 493-504, 2007.

NÄSLINDH-YLISPANGAR, A.; SIHVONEN, M.; KEKKI, P. Health, utilisation of health services, 'core' information, and reasons for nonparticipation: A triangulation study amongst non-respondents. Journal of Clinical Nursing, 17(22), p. 2972-2978, 2008.

NOONE, J. H.; STEPHENS, C. Men, masculine identities, and health care utilisation. Sociology of Health and Illness, 30(5), p. 711-725, 2008.

ROBINSON, M.; ROBERTSON, S. The application of social marketing to promoting men's health: A brief critique. International Journal of Men's Health, 9(1), p. 50-61, 2010.

SCHRAIBER, L. B.; FIGUEIREDO, W.; GOMES, R.; COUTO, M. T.; PINHEIRO, T. F.; MACHIN, R.; VALENÇA, O.

Necessidades de saúde e masculinidades: Atenção primária no cuidado aos homens. Cadernos De Saúde Pública 26(5), p. 961970, 2010.

SEPARAVICH, M. A.; CANESQUI, A. M. Saúde do homem e masculinidades na Política 
Nacional de Atenção Integral à Saúde do

Homem: Uma revisão bibliográfica. Saúde e

Sociedade, 22(2), p. 108-120, 2013.

SMITH, J. A.; BRAUNACK-MAYER, A., WITTERT, G.; WARIN, M. "I've been

independent for so damn long!":

Independence, masculinity and aging in a help seeking context. Journal of Aging

Studies, 21(4), p. 325-335, 2007. 